\title{
Melt-and-Mold Fabrication (MnM-Fab) of Reconfigurable Low-Cost Devices for Use in Resource-Limited Settings
}

Zhi Li ${ }^{b}$ Ian D. Tevis, ${ }^{a}$ Stephanie Oyola-Reynoso, ${ }^{a}$ Lucas B. Newcomb,${ }^{b}$ Julian HalbertsmaBlack, ${ }^{b}$ Jean-Francis Bloch, ${ }^{c}$ Martin Thuo ${ }^{a *}$

${ }^{a}$ Materials Science and Engineering Department, Iowa State University, 2220 Hoover Hall, Ames, IA 50011 USA

${ }^{b}$ Department of Chemistry, University of Massachusetts Boston, 100 Morrissey Blvd, Boston, MA 02125 USA

${ }^{c}$ Department of Paper, Print Media, and Biomaterials, Grenoble Institute of Technology, 461 Rue de la Papeterie, 38402 Saint-Martin-d'Héres, France

*Corresponding Author: Martin Thuo. Phone: +1(515)-294-8581.e-mail: mthuo@iastate.edu 


\begin{abstract}
Interest in low-cost analytical devices (especially for diagnostics) has recently increased; however, concomitant translation to the field has been slow, in part due to personnel and supplychain challenges in resource-limited settings. Overcoming some of these challenges requires the development of a method that takes advantage of locally available resources and/or skills. We report a melt-and-mold fabrication (MnM Fab) approach to low-cost and simple devices that has the potential to be adapted locally since it requires a single material that is recyclable and simple skills to access multiple devices. We demonstrated this potential by fabricating entry level bioanalytical devices using an affordable low-melting metal alloy, Field's metal, with molds produced from known materials such as plastic (acrylonitrile-butadiene-styrene (ABS)), glass, and paper. We fabricated optical gratings then $4 \times 4$ well plates using the same recycled piece of metal. We then reconfigured the well plates into rapid prototype microfluidic devices with which we demonstrated laminar flow, droplet generation, and bubble formation from T-shaped channels. We conclude that this MnM-Fab method is capable of addressing some challenges typically encountered with device translation, such as technical know-how or material supply, and that it can be applied to other devices, as needed in the field, using a single moldable material.
\end{abstract}

Keywords: Low-cost, bioanalytical, microfluidic, reconfigurable, green, reusable 


\section{Introduction}

The rapidly increasing global population faces threats of new diseases, scarcity of clean water, and, costs of living rising to crippling heights as global resources are strained to unmanageable limits.[1] This strain will have especially devastating consequences for developing/emerging nations.[1, 2] Providing adequate food, clean water, and healthcare is difficult because the necessary tools to diagnose diseases, measure nutrient content, detect food toxins, or assess the quality of water are not always available, especially in resource limited settings.[2, 3] The lack of reliable supply of good quality and efficient diagnostic tools in the developing world, is partly due to poor infrastructure (such as roads and bridges) necessary for uninterrupted supply. This breakdown in supply-chain of prefabricated disposable analytical devices makes it challenging - and expensive - to deliver care to populations in resource-limited settings who are also the most in need. The availability of cellular communication, however, has allowed for an influx of information, often free via the web, to almost every region of the world. New approaches to diagnosis and analytical measurements can therefore take advantage of the growing global connectivity and transfer some responsibility of device fabrication to those who need them most. This approach has been applied successfully in agricultural applications.[2, 4] It allowed the integration of better farming practices, such as strip farming, contour farming, and gabion construction, in many parts of rural Africa. Similarly, formal education and industrialization principles have been adopted in most parts of the world, not through material transfer, but predominantly through information sharing and local customization.

Recent developments in low-cost diagnostic and bio-analytical devices are driven, in part, by the availability of affordable materials coupled with low-cost fabrication techniques.[5] One example is the emergence of paper-based microfluidics ( $\mu$-pads) - an effort spearheaded by 
Whitesides and co-workers.[6-21] Although affordable wax-printing, 3D printing, and similar technologies that enhance the quality and complexity of paper-based devices are readily available in the developed world, they are almost non-existent in remote villages and other resource-limited environs.[10, 11, 18, 20,22] These low-cost diagnostic and bio-analytical devices have to overcome, and/or selectively exploit, the inherent hydrophilicity of paper to control aqueous bio-fluid movement.[6, 12, 20, 23] Paper technologies developed so far are often not adaptable for use in regions without electricity or where the requisite processing technology is unavailable. A practical technology that can be applied in these regions should not necessarily require grid-based electricity, should be simple, and can be accomplished with locally available resources (materials and personnel). Such restrictions are met by reliable and easy to understand techniques such as replica molding, by using reusable materials, applying simple sources of energy (e.g flames for heat) and globally available reagents like water. We believe that although the use of a low-cost, abundant material such as paper is desirable, other approaches are needed to introduce new materials in the development of low-cost, bio-analytical devices.

We therefore envision a new form of 'tele-medicine or tele-analysis' that involves the user as an active technology participant in not only running and interpreting the assay, or reporting the data over the cellular bandwidth but also in device fabrication. To achieve this goal, the requisite diagnostic and/or bio-analytical device(s) should; i) mainly be derived from locally available materials (abundant and reliable local supply), ii) use cheap (with respect to the immediate socio-economic setting) materials, iii) be easy to fabricate and use, iv) be powered using locally available resources, and v) be from an affordable reconfigurable material that can be reprocessed (see Supporting Information Table S1). This paper reports our initial development of analytical platforms/devices derived from soft, low-melting point alloys that are 
examples of reconfigurable, easy to process and re-use materials. We hypothesize that the use of low melting point $\left(\mathrm{T}_{\mathrm{m}}\right)$ metal(s) will allow the fabrication of various devices from the same piece of material using well established processes while utilizing some locally available resources for manpower and molds. To illustrate this capability, we fabricated basic bio-analytical devices such as well-plates, optical gratings, and microfluidic devices, from a re-usable metal (Figure 1). The high surface energy of the metal allows the use of both polar and non-polar solutions without affecting the fluid-flow behavior of the channel in microfluidic devices. To illustrate the adaptability of our approach, molds were made from plastics, glass, aluminum, and paper - all readily available materials even in the developing world.

Figure 1: Diagram of Melt-and-Mold Fabrication (MnM-Fab) of diffraction gratings, microfluidic devices, and well plates all using the same piece of low-melting metal and various molds.

\section{Materials and Methods:}

Materials: Bismuth indium tin (Field's metal, Bi/In/Sn at 32.5:51:16.5 wt\%, Alfa Aesar), bismuth tin eutectic (99.95\%, Bi/Sn at 58:42 wt \%, Alfa Aesar), indium (99.9\%, Alfa Aesar), and gallium (99.99\%, Strem Chemicals) were used as received. Polydimethylsiloxane molds (PDMS, Sylgard) were made by mixing the elastomer with the curing agent at a ratio of 10:1. Diffraction gratings with 1800 grooves $/ \mathrm{mm}(556 \mathrm{~nm}$ spacing) and 2400 grooves $/ \mathrm{mm} \mathrm{(417}$ nm spacing) were purchased from Edmund Optics and used as received. All other chemicals were obtained from Sigma-Aldrich and used as received.

General MnM Method: Field's metal, a low melting alloy, was melted using either a Bunsen burner or a laboratory heating gun in a ceramic crucible and poured over the molds at ambient conditions. For comparison purposes, we also fabricated devices from other low-melting 
metals (pure metals of bi- and tri- component alloys) to illustrate the versatility and simplicity of the MnM fab approach.

Fabrication of Well Plates Using Plastic Molds: Models of the desired well plate molds were created using SketchUp software, then molds were printed using a MakerBot Replicator 2X and an ABS filament. Molten Field's metal was then poured directly in the 3D-printed mold and left to cool at ambient conditions for at least $10 \mathrm{~min}$. The cooled metal easily separated from the plastic molds providing well-plates with an inner diameter of $7 \mathrm{~mm}$. Well-plates were filled with different polar and non-polar liquids of various volumes to demonstrate they can be used with either liquid in contrast to plastic well plates.

Fabrication of Gratings in Elastomeric Molds: The PDMS elastomer and curing agent were mixed at a ratio of 10:1. The desired diffraction grating was covered in the PDMS mixture, degassed, and then cured in an oven at $70{ }^{\circ} \mathrm{C}$ for 2 hours. The grating was then removed from the cured PDMS, leaving a negative of the original in the PDMS. The metal of choice (Field's metal, $\mathrm{Bi} / \mathrm{Sn}, \mathrm{Ga}$, or In) was heated until molten, then poured into the PDMS mold at ambient conditions. The metals were released from the molds after cooling to ambient conditions $(>10$ minutes). Negative replicas of plastic double diffraction gratings were made by pouring molten Field's metal over plastic diffraction gratings that had 13,500 lines/inch and pulled off after cooling to room temperature.

Light Diffraction by Replicated Gratings: A bright white light source was shone onto the molded gratings at ambient conditions. Diffracted light was directed onto a white background and the resulting diffraction pattern was photographed using a Nikon D7100 camera. 
Fabrication of Microfluidic Devices Using Paper Molds: Cardstock paper molds of the microfluidic device were created using a Cameo Silhouette craft cutter and accompanying software (similar molds were also made using scissors). The paper molds were then placed on a glass support, metallic fluid inlets were attached, and the re-molten Field's metal was poured onto the molds (with the inlets already positioned). The devices were allowed to cool at ambient conditions for $10 \mathrm{~min}$. The paper mold was then removed from the metal devices, which were subsequently immersed in an aqueous $1 \mathrm{M} \mathrm{H}_{2} \mathrm{SO}_{4}$ solution (95-98 \% sulfuric acid, SigmaAldrich) for 30 min to remove any organics introduced by the paper. In resource limited settings other dilute acids can be used such as muriatic acid or even dilute acetic acid in the form of vinegar. Warm vinegar can help clean off organics and also slightly etch the metal surface cleaning it further. Polyethylene tubing was connected to the hollow metal inlets and to a fluid delivery system. The devices were covered with 1 " $\times 3$ " microscope glass slides (treated with 1H,1H,2H,2H-perfluorooctyltriclorosilane vapor) using binder clips.

Operation of Microfluidic Devices: A syringe pump system (Fusion 200, Chemyx Inc.) was used to deliver hexadecane (Alfa Aesar, 99\%) dyed with Sudan Red 7B (Amresco), deionized water containing blue food coloring (McCormick) with 5\% Tween 20 (Amresco), or air. The rate of fluid delivery was controlled using the accompanying pump software. Device operation was documented with a Nikon D7100 camera.

Photography: All photographs were taken with a Nikon D7100 camera with either a Nikon DX AF-S Micro NIKKOR 85 mm lens or a Nikon DX VR AF-S NIKKOR 18-140 mm lens.

Atomic Force Microscopy (AFM) Measurement of Surface Parameters: Bruker Innova series AFM was used with a RTESPA probe in tapping mode to characterize the surface features 
of the gratings. Tapping mode was chosen to avoid disruption of molded surface features, thus preserving the native structure. Images obtained had scales from 3 to $20 \mu \mathrm{m}$ in width and length using the highest resolution available (1024 samples/line, scanning at $1 \mathrm{~Hz}$ ). All samples were scanned immediately after preparation to reduce the accumulation of surface contaminants. Each sample was scanned on the outermost and innermost regions to generate the most accurate representation of the entire surface.

Scanning Electron Microscopy (SEM): A Zeiss Supra55VP Field Emission SEM was used to examine the micro- and nanostructures of the Field's metal replica gratings and to determine the elemental composition of their surfaces. Samples were imaged and elements mapped using an electron beam accelerating voltage of $15 \mathrm{kV}$ and a working distance of $8.6 \mathrm{~mm}$. Images were collected using an Everhart-Thornley secondary electron detector. Elemental composition at various accelerating voltages was determined using an Energy Dispersive X-ray spectrometer with a silicon drift detector.

\section{Results and Discussion:}

Molds were manufactured from various materials (hard plastics (well-plates), elastomers (gratings), and paper (microfluidics)) to illustrate the versatility and adaptability of the Melt-andMold fabrication (MnM-fab) approach in device fabrication. MnM-Fab at its core is a replica molding technique and replica molding can quickly duplicate the shape, morphology and structure of a master typically with a polymer such as polydimethylsiloxane (PDMS) and polyurethane. The reliability of the technique is limited by van der Waals interactions and how well the material can fill the mold. Replicas can be made with features smaller than $100 \mathrm{~nm}$ without the need for advanced lithography techniques. [24] The lateral dimension of the smallest feature has been generated with a $30 \mathrm{~nm}$ resolution. [25] The usual material used in replica 
molding has been the elastomer PDMS but has problems with replicating high-aspect-ratio structures because of its small Young's Modulus. The resolution limit is set by the interplay between the replica's elasticity and the interfacial tension; smoothing will occur because of this tension even if the replica material fills the mold completely. [26] These problems may be overcome with surface modification and vacuum techniques but there are inherent limits to the replica molding technique.

Optical Gratings: Gratings are essential components in spectroscopy. The ability to separate white light into individual wavelengths, or into narrow bands, allows for quality spectroscopic signals and targeted measurements of specific compounds or molecular features like in colorimetric assays for determining water purity or detecting enzymes or their activity. Because metals are reflective, we hypothesized that a standard grating can be used as a mold template to produce similar features on a metal by replica molding. This molded grating can then be repurposed for other devices after spectral diffraction. Most gratings exhibit line spacing measuring in hundreds of nanometers (417 and $556 \mathrm{~nm}$ for those used in this study). Conformal flow of molten metals, especially non-Newtonian fluids, into molds with hundreds of nanometers separation can be limited by capillary forces[27], resulting in insufficient flow into these channels to provide the maximum level of light diffraction

Figure 1: Diffraction grating replication. (A) Diffraction pattern of white light on the patterned surface of an aluminum grating used as the parent mold. Diffraction of grating replication using (B) Ga, (C) In, (D) Bi:In:Sn, and (E) Bi:Sn. (F) AFM topography image of an indium-derived grating. (G) Side view of AFM topography of the original glass grating and an In-diffraction grating. $(\mathrm{H})$ SEM micrograph of Bi:In:Sn grating and EDS mapping of Sn and Bi content showing surface phase segregation.

To confirm our hypothesis, commercially available reflective diffraction gratings with 1800 grooves/mm and 2400 grooves/mm were purchased and used as supplied. For lowresolution applications, e.g., when preliminary results are needed, metal replicas may be 
sufficient for grating fabrication using a two-step MnM-fab procedure. To generate the mold, a commercially available grating was covered in liquid PDMS and then cured. The grating was released from the PDMS negative mold, leaving a grating-sized gap in the PDMS, with the negative diffraction grating pattern at the bottom. Molten liquid metals (either gallium, indium, Field's metal, and bismuth-tin) were each poured into the mold, cooled, and then released to give replica diffraction gratings. A white light was then used to illuminate the replicated surfaces and the diffraction patterns were projected onto a white surface and photographed (Figure 2).

The diffraction of white light by the original aluminum diffraction grating was strong, with clear and intense colors. The diffraction by the metal replicas was clearly visible, although it was relatively weaker in intensity and the colors were not as clearly resolved as by the original aluminum grating. The pure metals showed more intense and defined diffraction patterns compared to the metal alloys. The higher-melting temperature pure indium grating was selected for AFM topography analysis (Figure $2 \mathrm{f}$ and $2 \mathrm{~g}$ ). The replica gratings exhibited a step height of $8.0 \pm 1.0 \mathrm{~nm}$ with a $400 \pm 10 \mathrm{~nm}$ line spacing compared to the original mold with a step height of $85.7 \pm 6.6 \mathrm{~nm}$ and $530 \pm 20 \mathrm{~nm}$ line spacing. The regular spacing of the replica gratings was clear and closely matched that of the initial template in line spacing dimensions, even when considering the thermal contraction of the indium metal as it cools over $\sim 130{ }^{\circ} \mathrm{C}$. The major discrepancy seen in the replications was the trough depth; here the replicas were much shallower (low peak-to-valley dimensions) than the original template. Because the liquid metals do not readily wet the PDMS, we anticipated that capillary forces would limit penetration of the liquid metal into the line spacing of the PDMS mold. The replication of gratings with nano-sized features illuminates the possibilities of, and the challenges in, making other small functional devices like nano-channels for fluidic devices. Thermal contraction of the metal may also have 
reduced the step height, making these surfaces not as diffracting as the initial template. Dealloying and phase separation may also occur as the liquid metal alloys cool and solidify.[28] Visual inspection of the alloy replicas confirmed that they were not as smooth as the pure metal replicas. The scattered light during the diffraction experiment was also not as intense as seen for the pure metals (Figure $2 \mathrm{~d}$ and $2 \mathrm{e}$ ). This increase in surface roughness was most likely caused by phase-segregation of metals from the alloy melt during solidification of the grating - the metals have different thermal expansivities and change in volume on cooling. This surface phase segregation caused non-uniform diffraction patterns because the pure elements have different light reflectivity. SEM images of the Field's metal diffraction grating showed surface patchiness, i.e., the surfaces were either highly polycrystalline and/or composed of different elements. Typical Field's metal surfaces are rough because of phase separation (see Supporting Information Figure S1).[28] We confirmed the surface composition using energy dispersive Xray spectroscopy (EDS) elemental mapping, which showed clear phase separation of Sn and Bi corresponding to the different contrast patches seen in the SEM micrograph (Figure 2h).

Inexpensive plastic double diffraction gratings typically used for teaching the basic properties of light were also used as a mold for MnM-Fab. The plastic double diffraction grating with 13,500 lines/inch and $5.15 \pm 0.20 \mu \mathrm{m}$ peak-to-peak spacing was used to make a negative replica in Field's metal with $5.20 \pm 0.22 \mu \mathrm{m}$ trough-to-trough distance (See Supporting Information Figure S2. This close feature spacing size demonstrates how effective the technique can be on scales larger than $1 \mu \mathrm{m}$.

Indium melts at $157^{\circ} \mathrm{C}$, which may not be compatible with many organic-based mold materials. Gallium replicas exhibited the most intense and clear diffraction patterns. Gallium readily melts below $30^{\circ} \mathrm{C}$, hence a sorter thermal range for changes from liquid to solid. 
Although its low $\mathrm{T}_{\mathrm{m}}$ is convenient with respect to device fabrication, it poses challenges in operation, handling, and analysis. Besides its low $\mathrm{T}_{\mathrm{m}}$, gallium, as many metal melts, can significantly undercool making it difficult to fabricate devices using the MnM-fab approach.

Molding Well-Plates: Replica molding is a well-established technique used to manufacture devices and tools from soft materials such as plastics, metals, and alloys. Molding is simple, versatile, and easy to teach. Molds can be 3D-printed, or made from other readily available materials as long as they do not wet the material being molded. The high surface energy of most metals allows the mold to be made from almost any organic material. For multiwell plates, molds were made in 3D-printed ABS polymers as representatives of moldable thermoplastics. We also chose 3D printing first out of convenience (we had the printer at hand) and availability of thermoplastics inks (available in a developed world laboratory) such as ABS (acrylonitrile butadiene styrene), PLA (polylactic acid), and HIPS (high impact polystyrene) that can be molded using more traditional techniques. We used these molds to illustrate the use of thermoplastics in the MnM-fab process. The produced well plates may be used for cell culture studies, ELISA, or other diagnostic tests.

A 16-well positive relief ABS was printed on a Makerbot $2 X^{\circledR}$ 3D printer. Figure 3a shows a picture of the printed mold while the molding process is shown in Figure 3b. The molten metal, here Field's metal that had previously been molded into a diffraction grating, was poured directly onto the heat-stable ABS mold, creating a multi-well plate after solidification and lift-off (Figure 3c). Easy lift-off was possible because the metal contracts as it cools and solidifies, detaching from the mold. The replication was so effective that grooves generated during 3D printing were visible in the surface of the metal well-plates (Figures $3 \mathrm{c}$ and $3 \mathrm{~d}$ ). These grooves could be removed from the ABS mold by either mechanical grinding or by solvent smoothing 
using acetone.[20] It is also possible to grind/polish the metal well-plates post-replication to remove grooves or defects. A major advantage of this approach is that wells can be sized and shaped differently, allowing to perform multiple experiments with different volume requirements on the same plate and/or to reconfigure some of the wells (e.g., through hot-embossing) without interfering with other parts of the well-plate. Because these metals readily oxidize in air, generating a passivating oxide layer, the surface of the wells can be chemically modified to generate wells with varying surface properties on the same plate by exploiting the surface chemistry of the native oxide. Unlike conventional plastic-based well-plates, the reported wellplates can accommodate solutions irrespective of the polarity of the solvent with the exception of reactive species such as strong acids. The used well plates can be melted in boiling water to recover the metal while sterilizing it before remolding. One disadvantage of metal-based wellplates, however, is the potential corrosion of the metal, especially under acidic conditions. Depending on the surface composition, de-alloying may also occur. This reactivity can be mitigated through surface modification of the well-plates.

Figure 3: Metal well-plates made from 3D-printed plastic (ABS) molds. (A) A 3D-printed mold of a 16-well-plate. (B) Molten metal being poured onto mold. (C) Well-plate after casting, cooled and released from mold. (D) Wells filled with colored aqueous solutions.

Microfluidic Devices: Devices that can move and manipulate fluids at the micro- and nanoscale are utilized in particle synthesis, enzymatic analysis, proteomics, and diagnostics, among many other applications. The most commonly utilized material for microfluidic devices is PDMS, a siloxane-based elastomer. PDMS can be poured and gently cured at moderate temperatures to give a transparent and flexible device allowing it to be used for rapid prototyping. PDMS is permeable to many solvents, swells when exposed to certain chemicals, 
can selectively absorb components out of a solution, and can delaminate. Solid PDMS is a thermoset plastic, thus, it cannot be molded or reformed after it has cured.

In order to create a new paradigm using readily available mold materials and reusable device materials for rapid prototyping, we fabricated microfluidic devices using paper molds and low-melting, fusible, alloy(s). Paper molds were precisely cut using a Silhouette Cameo craft cutter, or a pair of scissors, in various mold designs including; 'T', 'U', '+', 'Y', '1' shapes. An example of a more complex paper mold can be seen in the Supporting Information (Figure S3). Molten recycled Field's metal (used to first make optical gratings then well-plates) was poured directly onto the paper molds, cooled and then released from the molds. The glass sheet provided a smooth and flat surface allowing the created metal device to be flat and smooth and hence be easily sealed. Occasionally, some paper fibers from the mold would get stuck in the narrow channels of the device, but they were easily removed with a pair of tweezers. Making paper molds is a cheap and fast way to create sacrificial molds. Steel needles were used as inlet connectors and a fluoroalkylsilane-treated glass slide was used to cover and seal the device using binder clips.

Figure 4: Imaging of the molded microfluidic channels. a) A wide-angle view of a channel as imaged by optical profilometry, some regions appear as holes because of the reflectivity of the metal towards the profilometry's laser. b) An image of a similar channel as shown in (a) obtained by low-resolution SEM. The channel appears well reproduced. c) Depth profile of a channel cross-section shows production of microscale channels within limits of the method and the errors due to reflectivity during depth measurements.

To demonstrate the reproducibility of the channels, we measured the depth and cross section profiles using contactless profilometry. The wide-angle 3D view of the microfluidic channels shows well defined walls and good reproducibility (Figure 4a). In the optical (contactless) profilometer, light reflection on the metal surface rendered some regions to appear 
rougher than they are (Figure 4a). To show continuity and calculate depth of the channels, we measured a line profile across the channel. Figure $4 \mathrm{c}$ provides the depth profile across the channel in Figure 4a. Due to imaging errors, the channels appear uneven and rough. Scanning electron microscopy images of the channels (Figure 4b) confirmed that the channels were much smoother than contactless (optical) surface profilometry indicated.

Figure 5: Microfluidic devices. (A) Paper-based molds for fabricating microfluidic devices using metal melts. (B) Schematic of the fabricated microfluidic device. (C) Flow device demonstrating laminar flow with aqueous solutions (water with a yellow or blue dye). (D) Laminar flow device that was reconfigured with extended channel using water (yellow and blue). Two additions (dotted lines) of different lengths were added to elongate the device shown in $(\mathrm{C})$.

Flow Properties in MnM-Fab Microfluidic Devices: Figure 5 shows a schematic and an example of a working T-device. Two dyed (blue and yellow) aqueous solutions were pumped into each inlet at the same rate to demonstrate that for low Reynolds number channels, as expected, laminar flow is observed (working "Y" and "U" devices can be seen in the Supporting Information Figure S4). The green color at the end of the channel was created by mixing of the liquids upon exiting the channel. If this device were made from PDMS it would have a set configuration and design that cannot be changed without fabricating a new device. Metal tools and components can, however, be joined and/or repaired by welding. Similarly, the metal microfluidic devices fabricated here can be altered without completely melting and remolding the entire device. For example, a functioning T-device was temporarily stopped and the channel length was extended by welding onto the end of the initial channel (Figure 5c) to give an elongated device (Figure 5d) that performed as though it was part of the original segment (see Supporting Information Figure S5). This example shows that the capabilities of an experimental device can be adjusted, as needed, by inserting add-ons of different designs or extending the 
length of a microfluidic channel, e.g., to improve mixing or separation of fluids. These devices are therefore graftable and reconfigurable.

\section{Functional Fluidic Performance of MnM-Fab Microfluidic Devices: Laminar flow in} microfluidic devices is one of many possible fluid applications of reconfigurable devices. Metal and metal alloys are resistant to many aqueous and organic solutions while also being impermeable. They can therefore be used to fabricate droplet and bubble generators. Here, droplet generators were created using the previously shown T-device with water and hexadecane as orthogonal solvents to give droplets. Since neither solvent wets the metal channels very well, droplets could be made from water (Figure 6A) or hexadecane (Figure 6C). Devices were also fabricated to make air bubbles in hexadecane (Figure 6B). In bubble formation, however, slight leakage of the carrier fluid into the air channel was detected. We operated these devices at varying flow rates to obtain droplets of regular sizes at different frequencies. As with other microfluidic devices, these droplet and bubble generators followed a simple scaling law (Equation 1).[20, 29] In conventional microfluidic devices, the carrier fluid and droplet behavior are dictated by the surface chemistry of the channel.[29] Because the surface energy of metals and metal alloys are much higher than PDMS $\left(\sim 550 \mathrm{~mJ} / \mathrm{m}^{2}\right.$ for bismuth and $\sim 20 \mathrm{~mJ} / \mathrm{m}^{2}$ for PDMS) [30, 31], and because metals do not swell in the presence of a solvent, we were able to reproducibly generate droplets from both hydrophilic and hydrophobic solvents.

$$
\frac{L}{W}=1+\alpha \frac{Q_{\text {droplet }}}{Q_{\text {carrier }}}
$$

where $L$ is the length and $W$ is the width of the droplet and is proportional to frequency (droplets/min), $Q_{d r o p l e t}$ and $Q_{\text {carrier }}$ are the flow rates of the droplet fluid and carrier fluid, respectively, and $\alpha$ is a constant that depends on the geometry of the junction. In this case the 
droplet fluid and carrier fluid are; i) water and hexadecane (Figure 6d), ii) hexadecane and air (Figure 6e), and, iii) hexadecane and water (Figure 6f). If the devices hold to the simple scaling law then the plot of droplets/min vs $Q_{\text {droplet }} / Q_{\text {carrier }}$ is linear (Figure 6).

Figure 6: Microfluidic devices derived from molded Field's metal showing droplet and bubble formation. (A) Tshaped droplet generator showing formation of hexadecane (red) droplets in water (blue), (B) air bubbles (clear) in hexadecane (red), and, (C) water (blue) droplets in hexadecane (red). The generators show a linear dependence of droplet frequency with the ratio of flow rates as shown in (D) hexadecane droplets, (E) air bubbles, and (F) water droplets.

Conclusion: We demonstrate that a low-melting point $\left(<100{ }^{\circ} \mathrm{C}\right)$ metal can be used to fabricate three different devices: optical grating, a well-plate and a microfluidic device. The performance of these devices is predictable based on the surface properties of the metal and they can be customized to fit different needs. This methodology, termed MnM-Fab, is a simple, yet powerful approach to mitigate problems with device availability/supply in resource-limited settings by using the devices and subsequently reusing the metal to mold new devices. This simple approach could significantly lower the cost of such devices and speed up the production of prototypes. By employing replica molding as the fabrication technique of choice, we believe that such devices can be fabricated almost anywhere using locally available mold materials while melting the metal using local energy sources like an open flame.

\section{ASSOCIATED CONTENT}

Supporting Information. This material is available free of charge via the Internet at http://pubs.acs.org.

\section{AUTHOR INFORMATION}




\section{Corresponding Author}

* Materials Science and Engineering, Iowa State University, 2220 Hoover Hall, Ames, IA 50011 USA. Email: mthuo@iastate.edu. Phone: +1-515-294-8581.

\section{Notes}

Authors declare no competing financial interests

\section{ACKNOWLEDGMENTS}

This work was supported by Iowa State University through startup funds and by the University of Massachusetts, Boston through undergraduate research fellowships to ZL and JHB. This work was performed in part using the facilities at the Harvard University's Center for Nanoscale Systems (CNS), a member of the National Nanotechnology Infrastructure Network (NNIN), which is supported by the National Science Foundation under NSF award PHY-0646094.

\section{REFERENCES}

[1] C. Juma, L. Yee-choong, Reinventing Global Health: The Role of Science, Technology, and Innovation, Lancet, 365 (2005) 1105-1107.

[2] C. Juma, Preventing Hunger: Biotechnology is Key, Nature, 479 (2011) 471-472.

[3] S. Al-Bader, H. Masum, K. Simiyu, A.S. Daar, P.A. Singer, Science-Based Health Innovation in SubSahara Africa, BMC International Health and Human Rights, 10 (2010) 1-9.

[4] R.E. Evenson, D. Gollin, Assessing the Impact of Green Revolution, 1960-2000, Science, 300 (2003) 758-762.

[5] D.M. Cate, J.A. Adkins, J. Mettakoonpitak, C. Henry, S.,, Recent dDevelopments in Paper-Based Microfluidic Devices, Langmuir, DOI (2014) ASAP.

[6] E. Carrilho, A.W. Martinez, G.M. Whitesides, Understanding Wax Printing: A Simple Micropatterning Process for Paper-Based Microfluidics, Analytical Chemistry (Washington, DC, United States), 81 (2009) 7091-7095.

[7] E. Carrilho, S.T. Phillips, S.J. Vella, A.W. Martinez, G.M. Whitesides, Paper microzone plates, Analytical Chemistry (Washington, DC, United States), 81 (2009) 5990-5998.

[8] C.-M. Cheng, A.W. Martinez, J. Gong, C.R. Mace, S.T. Phillips, E. Carrilho, K.A. Mirica, G.M. Whitesides, Paper-Based ELISA, Angewandte Chemie, International Edition, 49 (2010) 4771-4774, S4771/4771-S4771/4779.

[9] A.K. Ellerbee, S.T. Phillips, A.C. Siegel, K.A. Mirica, A.W. Martinez, P. Striehl, N. Jain, M. Prentiss, G.M. Whitesides, Quantifying Colorimetric Assays in Paper-Based Microfluidic Devices by Measuring the Transmission of Light through Paper, Analytical Chemistry (Washington, DC, United States), 81 (2009) 8447-8452. 
[10] W. Lan, E.J. Maxwell, C. Parolo, D. Bwambok, M.M. Thuo, A.B. Subramaniam, G.M. Whitesides, X.U. Zou, J. Hu, P. Buhlmann, M. Hamedi, Paper-based reference electrode and potentiometric ion sensing, in: USPTO (Ed.), (President and Fellows of Harvard College, USA; University of Minnesota). US, 2014. [11] X.-Y. Liu, M. Mwangi, X.-J. Li, M. O'Brien, G.M. Whitesides, Paper-based piezoresistive MEMS sensors, Lab on a Chip, 11 (2011) 2189-2196.

[12] A.W. Martinez, S.T. Phillips, M.J. Butte, G.M. Whitesides, Patterned paper as a platform for inexpensive, low-volume, portable bioassays, Angewandte Chemie, International Edition, 46 (2007) 1318-1320.

[13] A.W. Martinez, S.T. Phillips, E. Carrilho, S.W. Thomas, H. Sindi, G.M. Whitesides, Simple Telemedicine for Developing Regions: Camera Phones and Paper-Based Microfluidic Devices for RealTime, Off-Site Diagnosis, Analytical Chemistry (Washington, DC, United States), 80 (2008) 3699-3707. [14] A.W. Martinez, S.T. Phillips, Z. Nie, C.-M. Cheng, E. Carrilho, B.J. Wiley, G.M. Whitesides, Programmable diagnostic devices made from paper and tape, Lab on a Chip, 10 (2010) 2499-2504. [15] A.W. Martinez, S.T. Phillips, G.M. Whitesides, Three-dimensional microfluidic devices fabricated in layered paper and tape, Proceedings of the National Academy of Sciences of the United States of America, 105 (2008) 19606-19611.

[16] A.W. Martinez, S.T. Phillips, G.M. Whitesides, E. Carrilho, Diagnostics for the Developing World: Microfluidic Paper-Based Analytical Devices, Analytical Chemistry (Washington, DC, United States), 82 (2010) 3-10.

[17] A.W. Martinez, S.T. Phillips, B.J. Wiley, M. Gupta, G.M. Whitesides, FLASH: A rapid method for prototyping paper-based microfluidic devices, Lab on a Chip, 8 (2008) 2146-2150.

[18] Z. Nie, C.A. Nijhuis, J. Gong, X. Chen, A. Kumachev, A.W. Martinez, M. Narovlyansky, G.M. Whitesides, Electrochemical sensing in paper-based microfluidic devices, Lab on a Chip, 10 (2010) 477483.

[19] A.C. Siegel, S.T. Phillips, M.D. Dickey, N. Lu, Z. Suo, G.M. Whitesides, Foldable Printed Circuit Boards on Paper Substrates, Advanced Functional Materials, 20 (2010) 28-35.

[20] M.M. Thuo, R.V. Martinez, W.-J. Lan, X. Liu, J. Barber, M.B.J. Atkinson, D. Bandarage, J.-F. Bloch, G.M. Whitesides, Fabrication of Low-Cost Paper-Based Microfluidic Devices by Embossing or Cut-andStack Methods, Chemistry of Materials, 26 (2014) 4230-4237.

[21] G. Whitesides, X. Liu, X. Li, M. Thuo, M. O'Brien, Y. Sun, MEMS force sensors fabricated using paper substrates, USA, 2013, pp. 68pp.

[22] C. Zhao, M.M. Thuo, X. Liu, A microfluidic paper-based electrochemical biosensor array for multiplexed detection of metabolic biomarkers, Science and Technology of Advanced Materials, 14 (2013) 054402/054401-054402/054407.

[23] D.A. Bruzewicz, M. Reches, G.M. Whitesides, Low-Cost Printing of Poly(dimethylsiloxane) Barriers To Define Microchannels in Paper, Analytical Chemistry (Washington, DC, United States), 80 (2008) 3387-3392.

[24] Y.N. Xia, G.M. Whitesides, Soft lithography, Angewandte Chemie-International Edition, 37 (1998) 550-575.

[25] Y.N. Xia, E. Kim, X.M. Zhao, J.A. Rogers, M. Prentiss, G.M. Whitesides, Complex optical surfaces formed by replica molding against elastomeric masters, Science, 273 (1996) 347-349.

[26] O.D. Gordan, B.N.J. Persson, C.M. Cesa, D. Mayer, B. Hoffmann, S. Dieluweit, R. Merkel, On pattern transfer in replica molding, Langmuir, 24 (2008) 6636-6639.

[27] P.-G. de Gennes, F. Brochard-Wyart, D. Quere, Capillarity and Wetting Phenomena Drops, bubbles, Pearls, Waves, Springer, New York, 2004.

[28] S. Sengupta, H. Soda, A. McLean, Evolution of Microstructure in Bismuth-indium-tin Eutectic Alloy, Journal of Materials Science, 40 (2005) 2607-2610. 
[29] P. Garstecki, M.J. Fuerstman, H.A. Stone, G.M. Whitesides, Formation of droplets and bubbles in a microfluidic T-junction-scaling and mechanism of break-up, Lab on a Chip, 6 (2006) 437-446.

[30] J.E. Mark, Physical Properties of Polymers Handbook, Springer New York2007.

[31] A. Singh, A.P. Tsai, Melting behaviour of lead and bismuth nano-particles in quasicrystalline matrix The role of interfaces, Sadhana-Academy Proceedings in Engineering Sciences, 28 (2003) 63-80. 


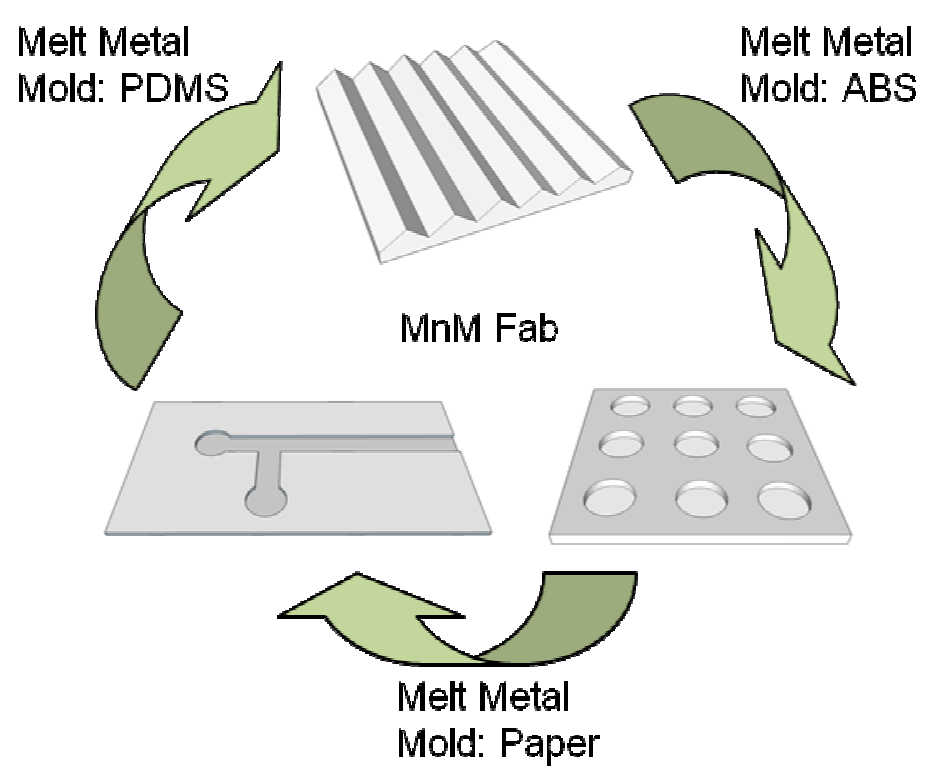

Figure 1: Diagram of Melt-and-Mold Fabrication (MnM-Fab) of diffraction gratings, microfluidic devices, and well plates all using the same piece of low-melting metal and various molds. 

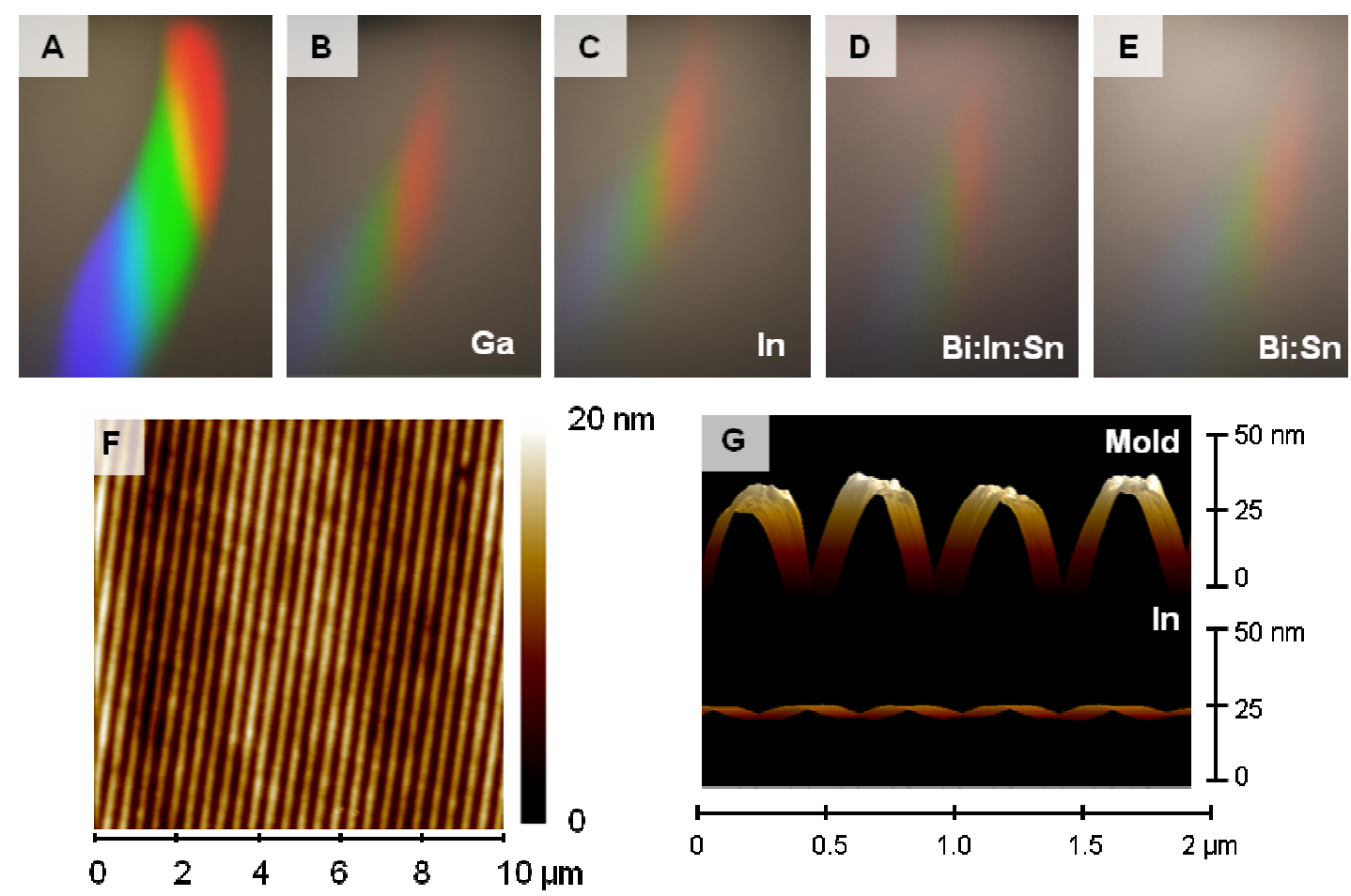

$20 \mathrm{~nm}$
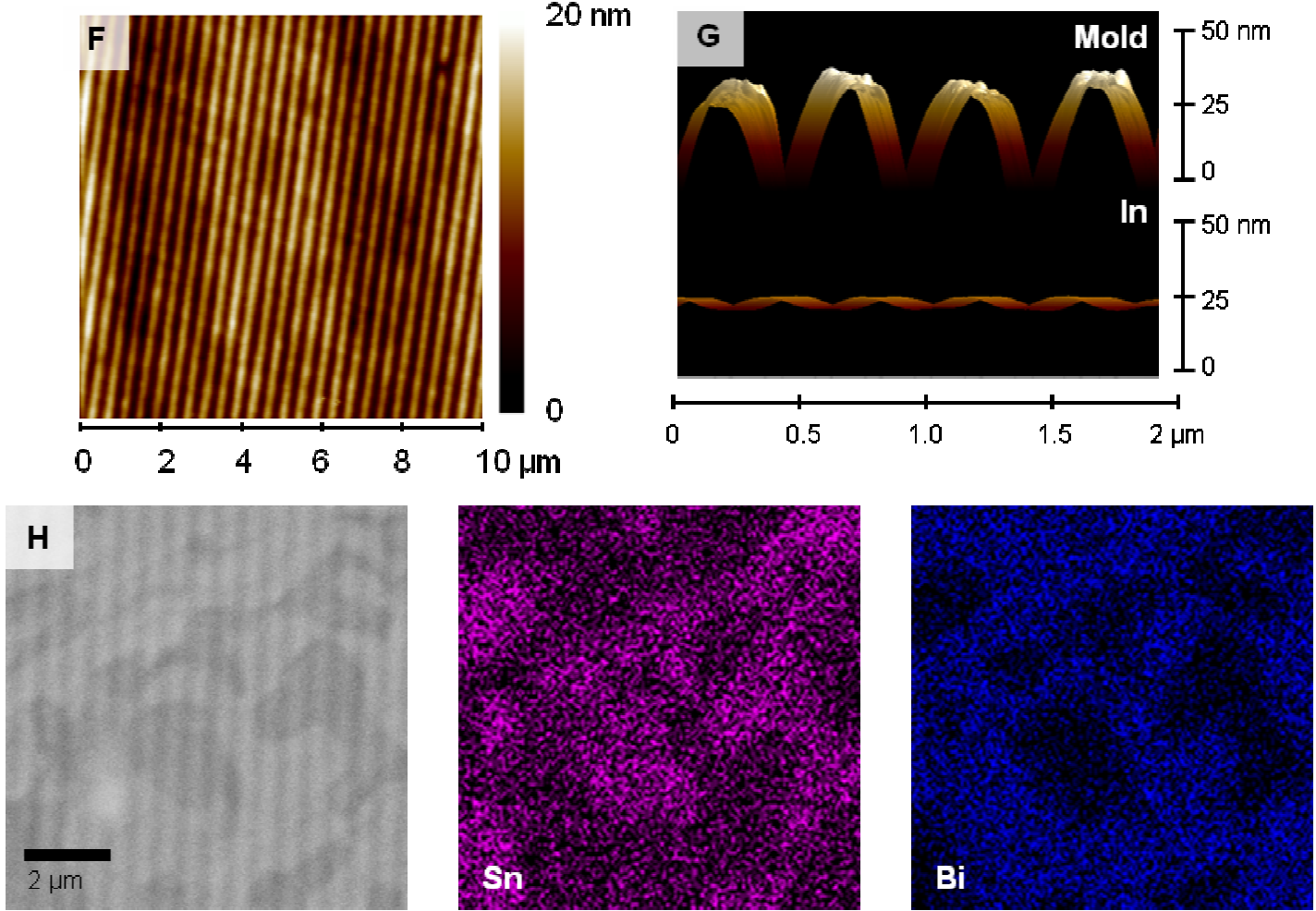

Figure 2: Diffraction grating replication. (A) Diffraction pattern of white light on the patterned surface of an aluminum grating used as the parent mold. Diffraction of grating replication using (B) Ga, (C) In, (D) Bi:In:Sn, and (E) Bi:Sn. (F) AFM topography image of an indium-derived grating. (G) Side view of AFM topography of the original glass grating and an In-diffraction grating. (H) SEM micrograph of Bi:In:Sn grating and EDS mapping of $\mathrm{Sn}$ and Bi content showing surface phase segregation. 


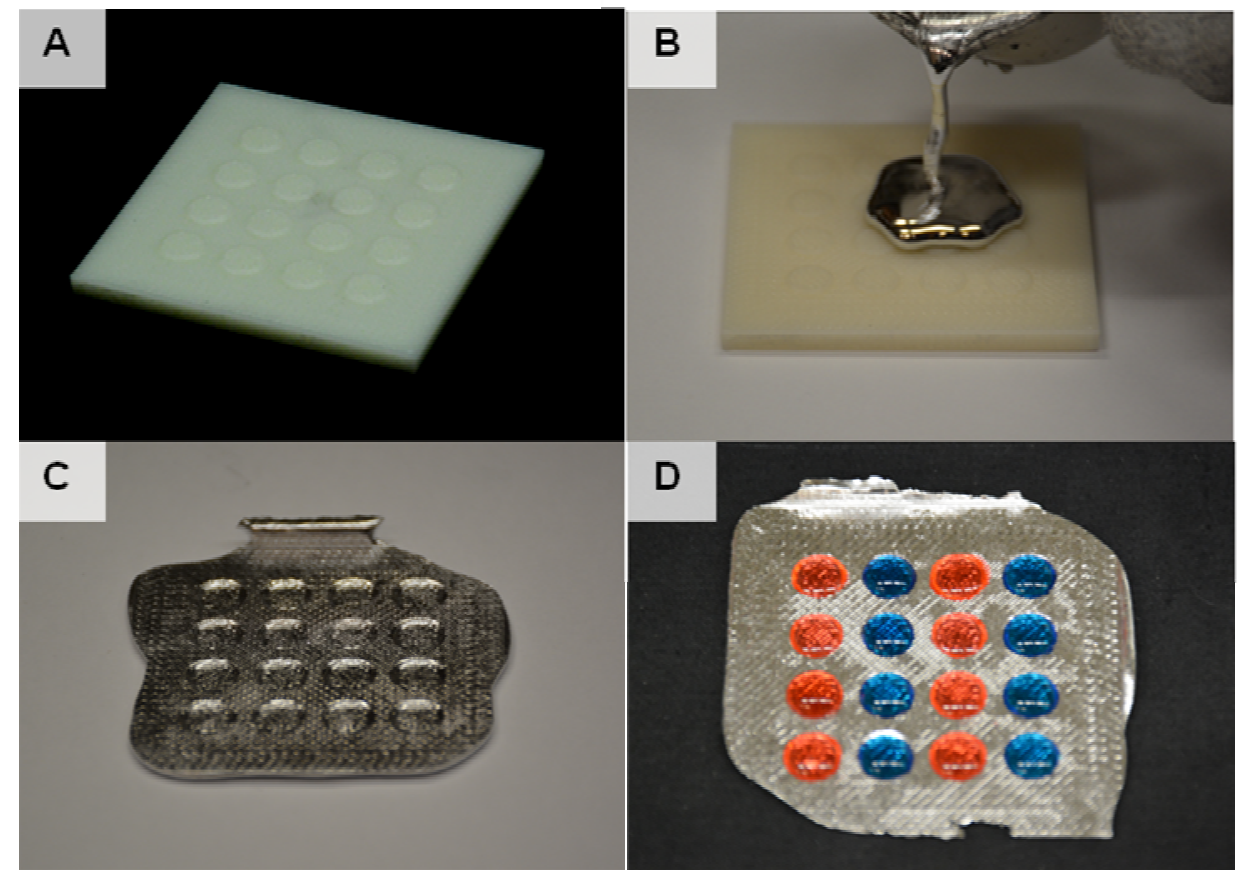

Figure 3: Metal well-plates made from 3D-printed plastic (ABS) molds. (A) A 3D-printed mold of a 16-well-plate. (B) Molten metal being poured onto mold. (C) Well-plate after casting, cooled and released from mold. (D) Wells filled with colored aqueous solutions. 

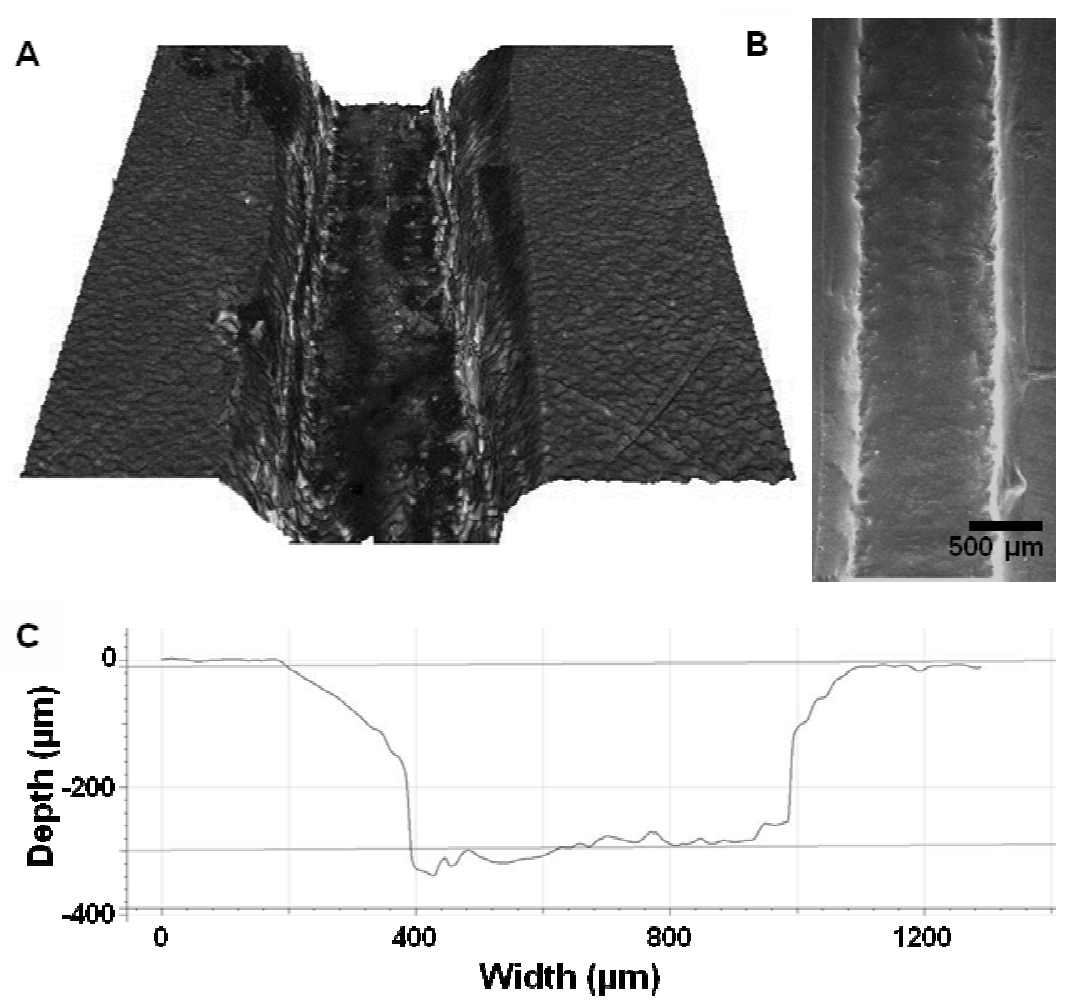

Figure 4: Imaging of the molded microfluidic channels. a) A wide-angle view of a channel as imaged by optical profilometry, some regions appear as holes because of the reflectivity of the metal towards the profilometry's laser. b) An image of a similar channel as shown in (a) obtained by low-resolution SEM. The channel appears well reproduced. c) Depth profile of a channel cross-section shows production of microscale channels within limits of the method and the errors due to reflectivity during depth measurements. 


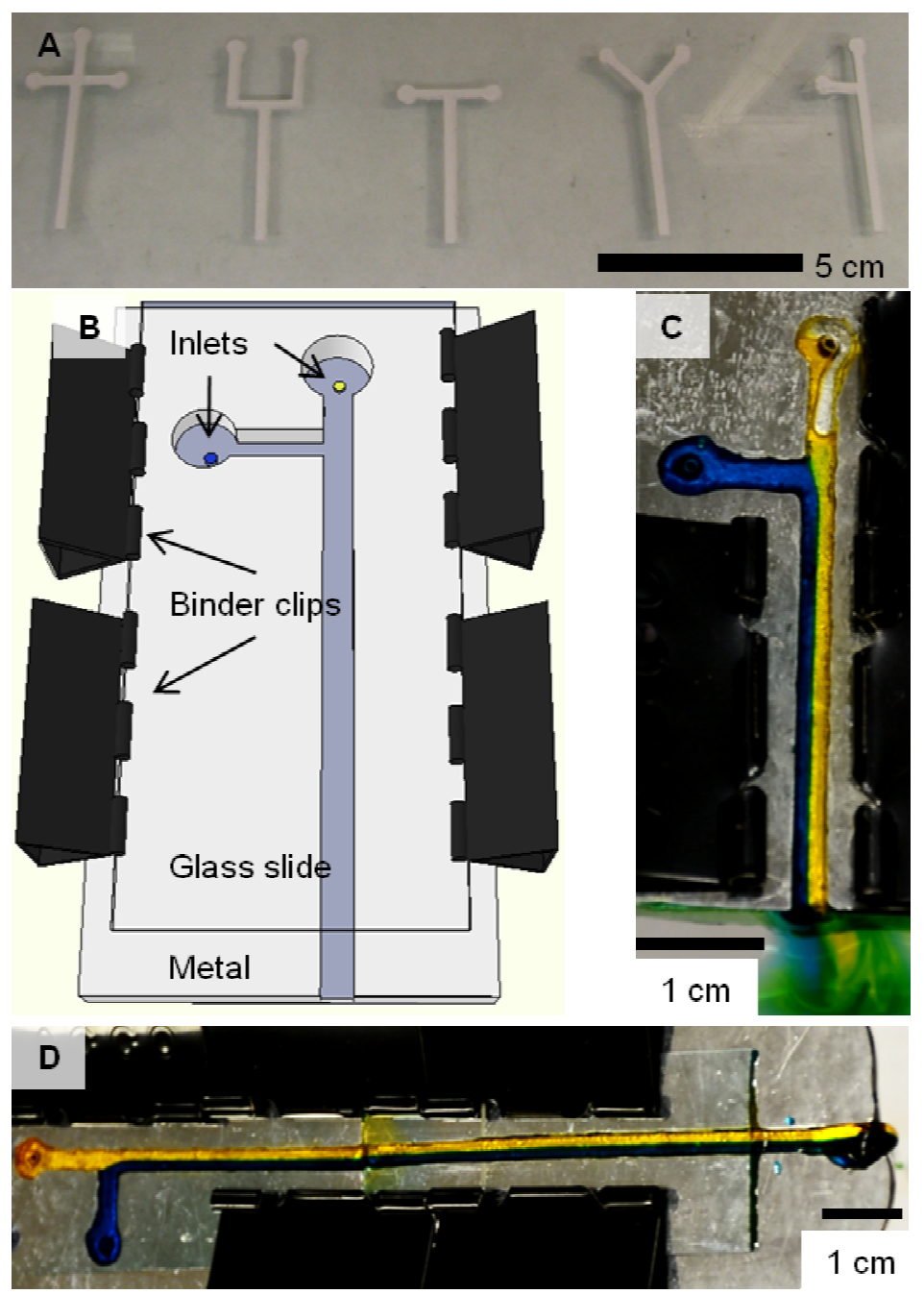

Figure 5: Microfluidic devices. (A) Paper-based molds for fabricating microfluidic devices using metal melts. (B) Schematic of the fabricated microfluidic device. (C) Flow device demonstrating laminar flow with aqueous solutions (water with a yellow or blue dye). (D) Laminar flow device that was reconfigured with extended channel using water (yellow and blue). Two additions (dotted lines) of different lengths were added to elongate the device shown in $(\mathrm{C})$. 
A

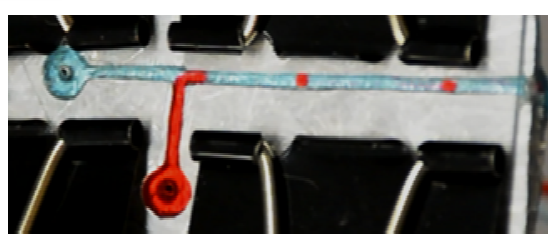

B

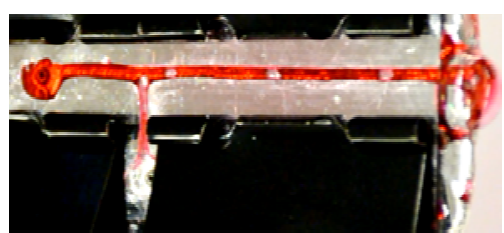

D

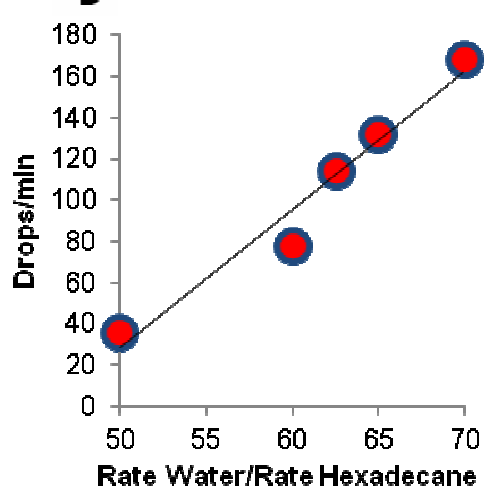

$\mathbf{E}$

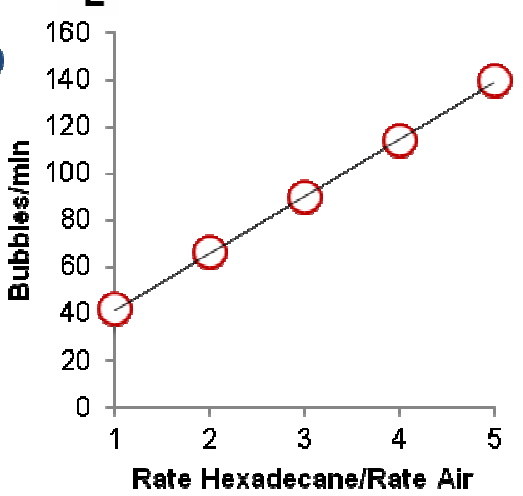

C
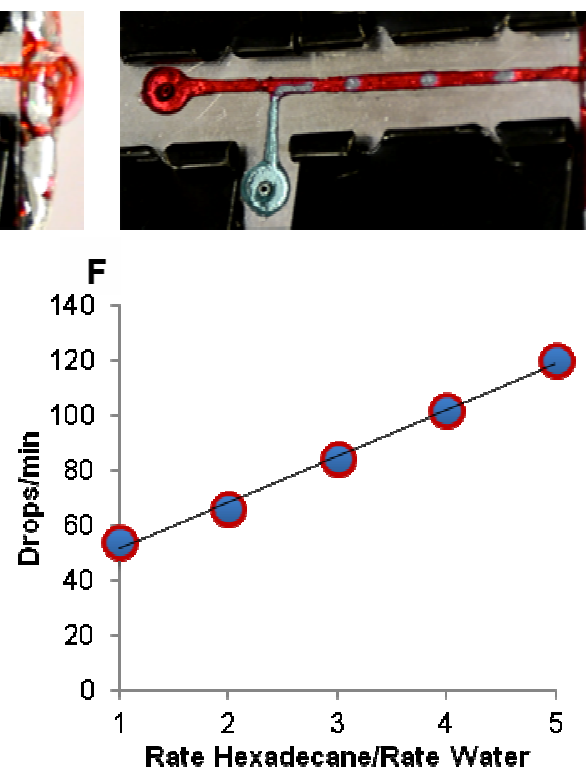

Figure 6: Microfluidic devices derived from molded Field's metal showing droplet and bubble formation. (A) Tshaped droplet generator showing formation of hexadecane (red) droplets in water (blue), (B) air bubbles (clear) in hexadecane (red), and, (C) water (blue) droplets in hexadecane (red). The generators show a linear dependence of droplet frequency with the ratio of flow rates as shown in (D) hexadecane droplets, (E) air bubbles, and (F) water droplets. 

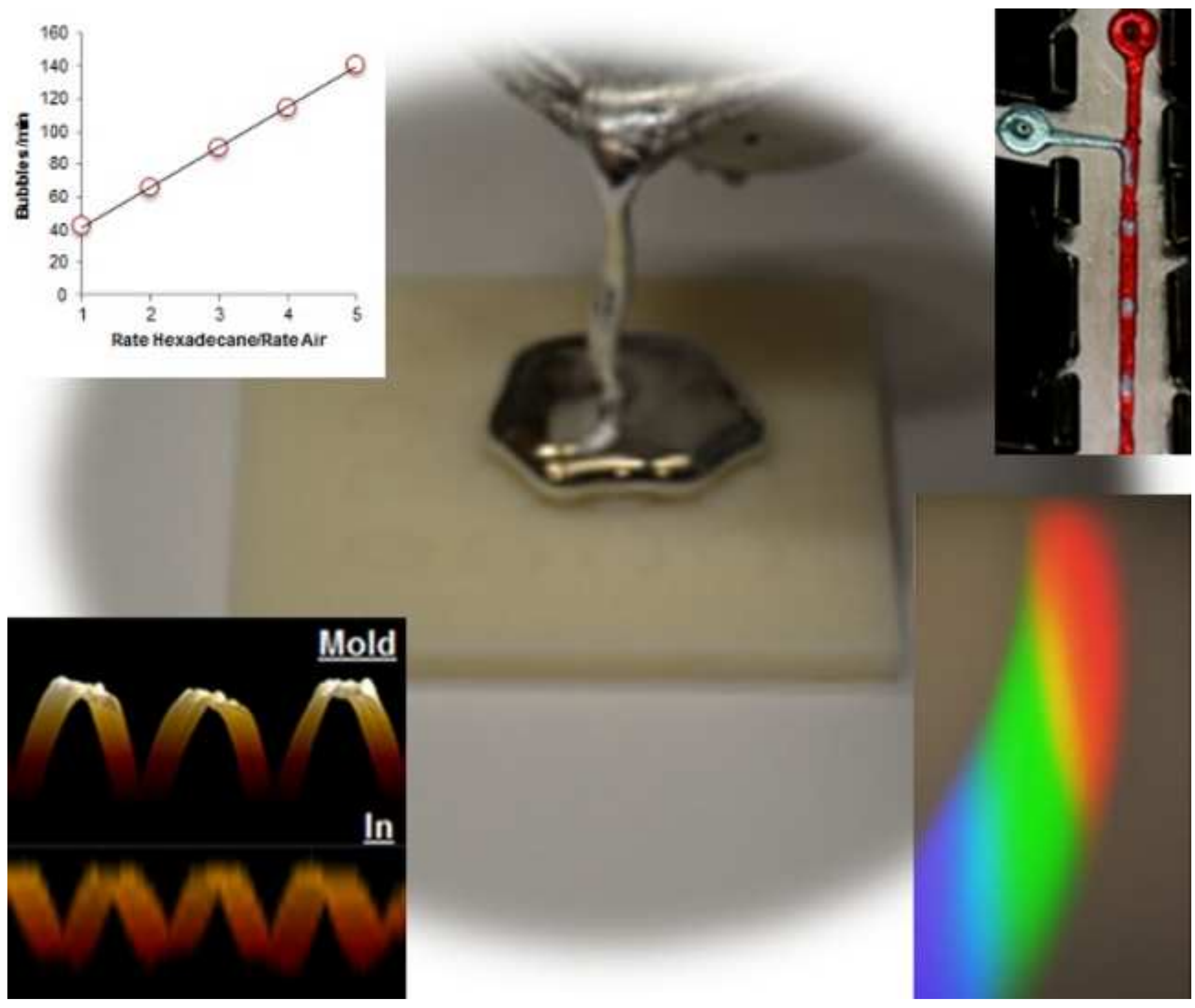

In 